\title{
Die Abkürzungsseuche
}

- Als Physiotherapeutin kenne ich sie alle: die ADLs, PNFs und AHBs der Physiowelt. Sie erleichtern uns die Kommunikation, wenn wir über den Klinikflur hechtend noch schnell die wichtigsten Fakten mit unseren Kollegen austauschen. „Frau M. von S13, seit gestern MRSA, VAS 8, ROM: LWS-Flex $30^{\circ}$ - bitte AP-Mobi, morgen geht's in die AHB." Doch seien wir ehrlich: Nicht nur gegenüber unseren Kollegen benutzen wir die Abkürzungen, auch in Arztbriefen, Kassenanschreiben und, wenn wir nicht aufpassen, im Gespräch mit unseren Patienten.

_ Gestern klagte mir eine befreundete Ärztin ihr Leid. Sie ist seit 20 Jahren Hausärztin und mit allen Wassern gewaschen. „Ihr erwartet, dass wir eure Berichte lesen - dann schreibt

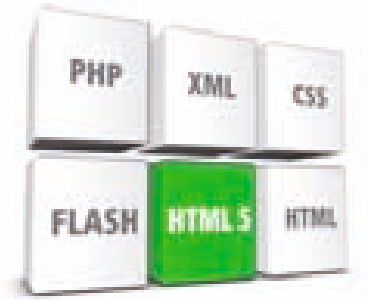
sie bitte auch so, dass ich sie verstehe." Ich hakte nach, und siehe da, am Morgen war sie über die Zeile „bzgl. ADL vollst. selbstst." gestolpert. Brust raus und Verteidigungshaltung eingenommen: „ADL, das weiß jeder im Gesundheitswesen“, behauptete ich selbstbewusst. „Nein“, anwortete sie genervt, ,und im Internet fand ich die Unternehmensberatung Arthur D. Little. Ein vollständig selbstständiger Unternehmensberater war der Patient aber nicht." Auch Anti-Dekubitus-Lagerungssysteme und die Antidiffamierungsliga schienen ihr nicht die richtige Erklärung. Erst Wikipedia brachte Aufschluss. Als vierten Punkt fand sie dort „Activities of daily living, siehe Aktivitäten des täglichen Lebens“.

_ Eine Geschichte, die ähnlich sicherlich tausendfach vorkommt. Die wenigsten machen sich aber wie diese gewissenhafte Ärztin die Mühe, unsere Abkürzungen $z u$ recherchieren. Wollen wir gehört werden, sollten wir verständlich bleiben! Im Alltag begegnen uns schließlich auch ständig Kürzel, die uns in den Wahnsinn treiben. Oder wussten Sie, dass PLZ in einer SMS für „bitte“ steht?

In diesem Sinne: $M C$ und $H N Y$

(Frohe Weihnachten und ein glückliches Neues Jahr!)

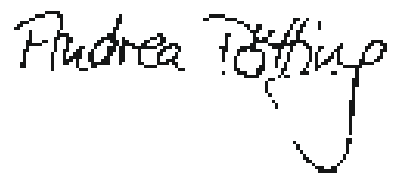

PS: Zurzeit begleiten mich ganz neue Abkürzungen im Alltag. SSW, SL und APGAR kannte ich aus der Ausbildung, aber bei BPD, FOD und APD - alles zu finden in einem Mutterpass - musste ich recherchieren. Mal sehen, ob sich das während meines MuSchu im nächsten Jahr ändert...
Die Abkürzungsseuche kursiert in vielen Branchen. Wollen wir verstanden werden, sollten wir sie aktiv bekämpfen.

\section{PHYSIOBONUS}

physiobonus heißen die Gewinnspiele in physiopraxis. Wer teilnehmen möchte, nutzt das Formular unter www.thieme.de/ physioonline in der Rubrik physioexklusiv. Und das gibt es in dieser Ausgabe zu gewinnen:

\section{Kursplatz}

1 Kursplatz „Manuelle Reflextherapie - Technikkurs 1 “ Seite 54

1 Kurs nach Wahl („Schmerzen verstehen“ oder „Funktionelle Neuroanatomie der Motorik") Seite 76

Bücher

2-mal „Checkliste Viszerale Osteopathie“ Seite 42 3-mal „Multitasking“ Seite 58 3-mal „physiolexikon“ Seite 97

und außerdem DVD „Beweg dich einfach“ Seite 27 3 „Winter-Wohlfühlpakete“ Seite 97

2 Polar-Pulsuhren Seite 97 